\title{
Exfoliation of Graphite with Triazine Derivatives under Ball-Milling Conditions: Preparation of Few-Layer Graphene via Selective Noncovalent Interactions
}

\author{
Verónica León, ${ }^{\dagger, \ddagger}$ Antonio M. Rodriguez, ${ }^{\dagger}$ Pilar Prieto, ${ }^{\dagger}$ Maurizio Prato, $^{\ddagger}$ and Ester Vázquez ${ }^{\dagger, *}$ \\ †Departamento de Química Orgánica, Facultad de Ciencias y Tecnologías Químicas-IRICA, Universidad de Castilla-La Mancha, 13071 Ciudad Real, Spain and \\ ${ }^{\ddagger}$ Department of Chemical and Pharmaceutical Sciences, University of Trieste, Piazzale Europa 1, 34127 Trieste, Italy
}

ABSTRACT A ball-milling treatment can be employed to exfoliate graphite through interactions with commercially available melamine under solid conditions. This procedure allows the fast production of relatively large quantities of material with a low presence of defects. The milling treatment can be modulated in order to achieve graphene flakes with different sizes. Once prepared,

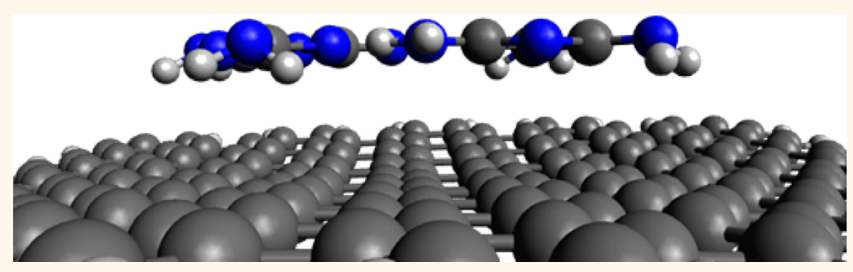
the graphene samples can be redispersed in organic solvents, water, or culture media, forming stable dispersions that can be used for multiple purposes. In the present work, we have screened electron-rich benzene derivatives along with triazine derivatives in their respective ability to exfoliate graphite. The results suggest that the formation of a hydrogen-bonding network is important for the formation of multipoint interactions with the surfaces of graphene and that can be used for the exfoliation of graphite and the stabilization of graphene in different solvents. Aminotriazine systems were found to be the best partners in the preparation and stabilization of graphene layers in different solvents, while the equivalent benzene derivatives did not show comparable exfoliation ability. Computational studies have also been performed to rationalize the experimental results. The results provide also the basis for further work in the preparation of noncovalently modified graphene, where derivatives of aminotriazines can be designed to form extensive hydrogen-bond 2D networks on the graphene surface with the aim of manipulating their electronic and chemical properties.

KEYWORDS: triazine $\cdot$ graphene $\cdot$ noncovalent interactions $\cdot$ ball milling $\cdot$ density functional calculations

chemical vapor deposition (CVD) methods produce, in general, graphene with a low number of defects that can be very useful for highly demanding electronic applications, in which small quantities of graphene are still sufficient. ${ }^{4}$ However, for the development of large-scale applications, ranging from conductive inks and fillers in composites to sensors or batteries, cost-effective production methods would be advisable with a good balance between ease of fabrication and/or manipulation and preservation of graphene properties. Moreover, besides biomedical applications, ${ }^{5}$ considering that graphene could be integrated into new electronics or composites, it would be fundamental to evaluate the impact of this new material on health and environment. ${ }^{6,7}$ All these studies require graphene dispersions *Address correspondence to
ester.vazquez@uclm.es.

Received for review October 3, 2013 and accepted December 31, 2013.

Published online 10.1021/nn405148t

() XXXX American Chemical Society leading to materials with different properties, suitable for diverse applications. ${ }^{3}$ The 
<smiles>Nc1nc(N)nc(N)n1</smiles>

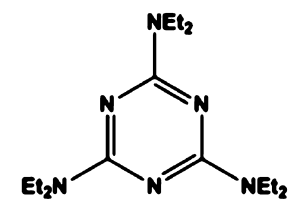

2,4,6-triamino-1,3,5-triazine 2,4,6-tri(N,N-diethylamino)-1,3,5-triazine (Melamine) (TNE-T)

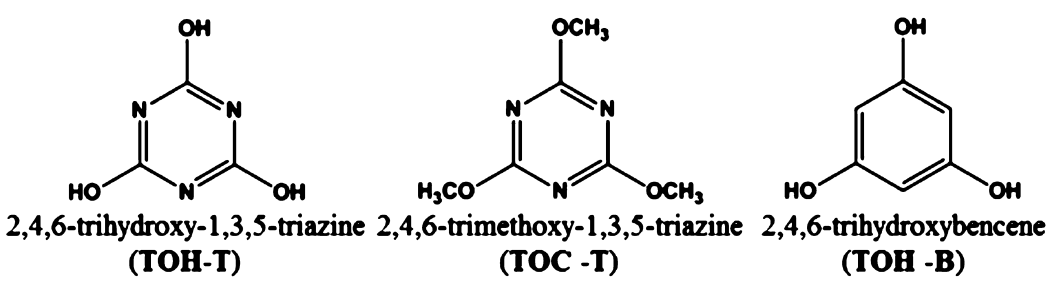

Chart 1. Chemical Structure of the Exfoliating Agents Used in This Study with Their Names and Corresponding Acronyms As Used in the Text

in aqueous media and controlled chemical modification. $^{8}$

In principle, exfoliation from bulk graphite is the most economical way to achieve large quantities of graphene. In addition, liquid-phase exfoliation techniques ${ }^{9}$ present several advantages because stable suspensions of graphene can be used for various processing steps of the material such as film deposition, surface modification, and chemical functionalization. The exfoliation of graphene into solution requires breaking the enormous van der Waals-like forces between graphite layers, which can be achieved by sonication of graphite in solvents ${ }^{10}$ and by chemical and electrochemical oxidation. ${ }^{11}$ However, these techniques also have some limitations. Oxidation techniques are very effective but disruptive and, even after reduction, produce high defective graphene. ${ }^{12}$ Sonication in organic solvents gives graphene of better quality, though still defective, but usually the flake size is relatively small.

Moreover, graphene layers tend to aggregate in order to re-establish the graphitic structure and to minimize surface free energy. This can be avoided by covalent functionalization ${ }^{13,14}$ or by noncovalent interaction with stabilizers, such as surfactants, polymers, and aromatic molecules. ${ }^{15}$ Though covalent techniques may alter significantly the electronic structure of graphene, the absorption of molecules on graphene can serve as a mere protecting coat of the graphene sheets. In other cases, the adsorption of certain molecules can also induce a band gap in graphene. ${ }^{16}$ Thus, the understanding of noncovalent interactions of different molecules with graphene is a very interesting topic for the development of new derivatives for desirable applications, including graphene-based chemical detectors, field-effect transistors, or organic optoelectronic devices.

Recently, we described an interesting alternative for the preparation of stable dispersions of graphene in different solvents, driven by an easy and eco-friendly ball-milling approach. ${ }^{17}$ Mechano-chemical activation of carbon nanostructures has generated great interest in recent years, ${ }^{18}$ and it has lately been used for the selective functionalization of graphene nanoplatelets. ${ }^{19}$ In our work, we used a ball-milling treatment to exfoliate graphite through interactions with commercially available melamine under solid conditions. This procedure allows the fast production of relatively large quantities of material with a low presence of defects. The milling treatment can be modulated in order to achieve graphene flakes with different sizes. Once prepared, the graphene samples can be redispersed in organic solvents, water, or culture media, forming stable dispersions that can be used for multiple purposes.

There are several interesting aspects in the use of melamine as dispersing agent in ball-milling experiments. First of all, melamine can be easily washed away, leaving pure graphene flakes in organic or water dispersions. Another question relates to the size of melamine, which is a small molecule, compared with the structure of polyaromatic hydrocarbons used for the exfoliation of graphite. In order to explain these results and especially to understand the role of melamine in the exfoliation, we have performed further experimental and computational investigations. We have modified the structure of the exfoliating agent from melamine to other triazine/benzene derivatives. This study aims to respond to many fundamental questions: Is this exfoliating process only induced by melamine? Can we use other triazine systems to obtain the exfoliation and, at the same time, stabilize graphene flakes in different solvents? Does this work disclose new concepts for the noncovalent modifications of graphene?

\section{RESULTS AND DISCUSSION}

Experiment. The compounds depicted in Chart 1 were commercially available, except 2,4,6-tri $(\mathrm{N}, \mathrm{N}$ diethylamino)-1,3,5-triazine, which was prepared following published procedures. ${ }^{20}$ We have used a ball-milling 


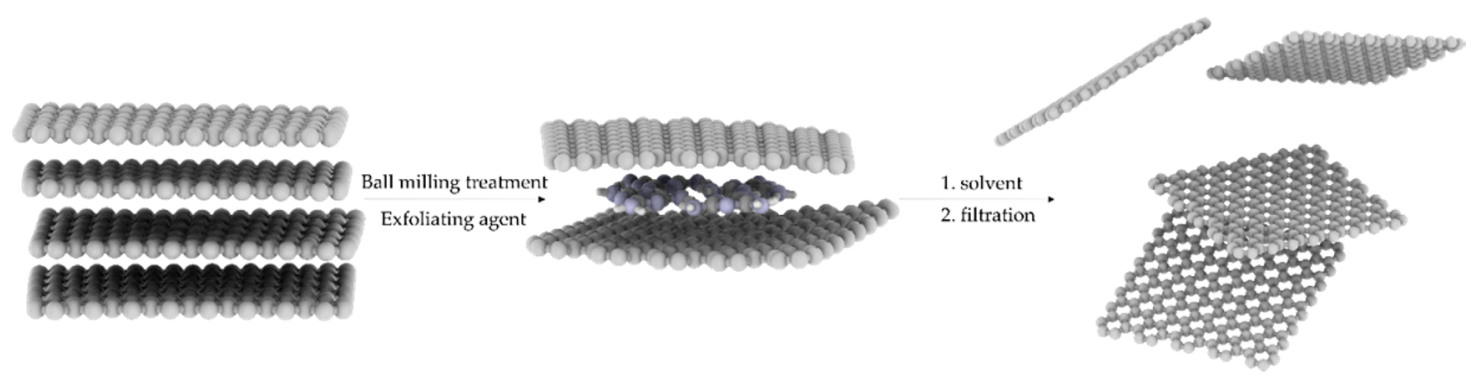

Scheme 1

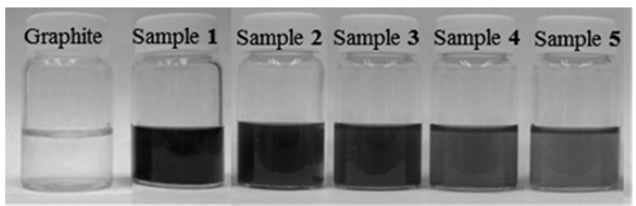

Figure 1. Photograph of graphite and graphene/exfoliating agent solutions in DMF.

TABLE 1. Comparison of Effectiveness of Exfoliating Agent as Stabilizer of Graphene in DMF

\begin{tabular}{ccccc} 
sample & agent & $\begin{array}{c}\text { concentration of } \\
\text { exfoliating agent } \\
(\mathbf{m g} / \mathbf{m L})^{\boldsymbol{a}}\end{array}$ & $\begin{array}{c}\text { concentration of } \\
\text { graphene } \\
(\mathbf{m g} / \mathbf{m L})^{\boldsymbol{a}}\end{array}$ & $\begin{array}{c}\text { graphene concn/ } \\
\text { exfoliating agent } \\
\text { concn }\end{array}$ \\
\hline $\mathbf{1}$ & melamine & 1.13 & 0.37 & 0.33 \\
$\mathbf{2}$ & TNE-T & 2.28 & 0.12 & 0.05 \\
$\mathbf{3}$ & TOH-T & 0.98 & 0.07 & 0.07 \\
$\mathbf{4}$ & TOC-T & 1.33 & 0.05 & 0.04 \\
$\mathbf{5}$ & TOH-B & 0.95 & 0.05 & 0.05 \\
\hline
\end{tabular}

${ }^{a}$ Calculated by thermogravimetric analysis.

approach to exfoliate graphite through interactions with different 1,3,5-triazine/benzene derivatives (Chart 1) under solid conditions (Scheme 1). In a typical procedure, $7.5 \mathrm{mg}$ of graphite and $0.16 \mathrm{mmol}$ of the triazine/benzene derivative were ball-milled at $100 \mathrm{rpm}$ during $30 \mathrm{~min}$ under air atmosphere. After the milling treatment, the resulting solid mixtures were dispersed in $20 \mathrm{~mL}$ of water or DMF to produce black suspensions. After letting the solutions rest for 5 days, the precipitate was removed, and the resulting dispersions were stable at room temperature within weeks. A comparison between the dispersions obtained in DMF, using the different exfoliating agents, is presented in Figure 1 and Table 1. No dispersion but only a black solid was obtained when graphite was ball-milled in the absence of exfoliating agent.

Typical X-ray diffraction (XRD) patterns of solid samples before and after ball-milling treatment with melamine are reported in Figure 2, showing that the sharp graphitic (002) reflection around $25^{\circ}$ clearly decreases after the milling.

Thermogravimetric analysis (TGA) provides insights into the composition of graphite and the exfoliating agent after the ball-milling process (Supporting Information, Figure S1). Graphite is thermally stable when heated up to $900^{\circ} \mathrm{C}$ under inert atmosphere. Samples

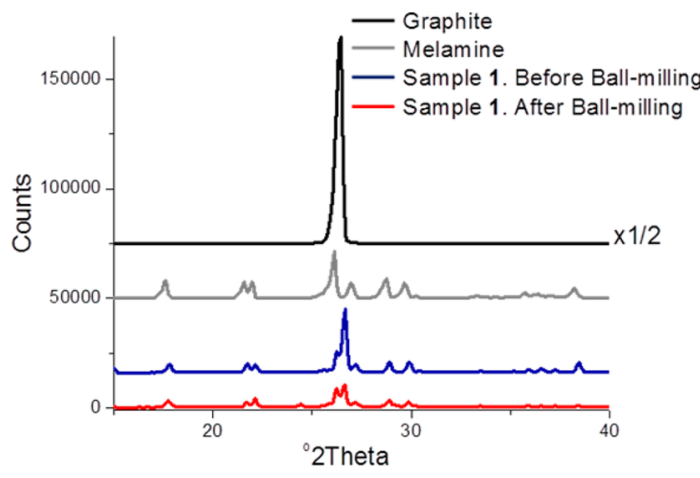

Figure 2. XRD patterns of graphite, melamine, and sample 1 before and after the ball-milling treatment.

of graphite after ball-milling treatment with the triazine/benzene derivatives present a weight loss at around $300{ }^{\circ} \mathrm{C}$, which corresponds to the amount of exfoliating agent used in each sample. No further loss takes place above or below this temperature, showing that no oxidative defects have been generated around the graphite flakes. ${ }^{21}$ TGA (Figure S2) has also been used to calculate the concentration of pure exfoliating agent and graphene in dispersions of DMF (Table 1) and water (see Supporting Information, Table S1).

The final concentration of graphene in DMF depends on the substituents in the triazine derivatives. The highest graphene concentration is obtained using melamine; in fact, the graphene to melamine ratio is higher than all the tested compounds and is higher than the one described for other polymers and surfactants. ${ }^{15}$ While the possibility of forming hydrogen bonds between molecules leads to higher graphene concentrations (1 vs $\mathbf{2}$ and $\mathbf{3}$ vs $\mathbf{4}$ in Table 1), amino substituents in the triazine rings lead to the highest graphene concentrations (entries $\mathbf{1}$ and $\mathbf{2}$ vs $\mathbf{3}$ and $\mathbf{4}$ in Table 1), which suggests that amino substituents provide more stabilization than hydroxyl groups. Water dispersions can also be prepared (Table S1, Supporting Information), in which case the solubility of the exfoliating agent seems to be the key factor in the stabilization of the graphene layers: derivatives such as 2,4,6trihydroxy-1,3,5-triazine, with a very high solubility in water, do not disperse the graphene flakes in water.

The as-prepared dispersions can be filtered and washed to remove the triazine/benzene derivatives, and the graphene samples can then be redispersed in 
TABLE 2. Final Graphene Concentration, In-Plane Crystallite Sizes $\left(L_{a}\right)$, and Distance between Defects $\left(L_{D}\right)$ of $G r a p h e n e$ Samples Prepared Using Different Exfoliating Agents

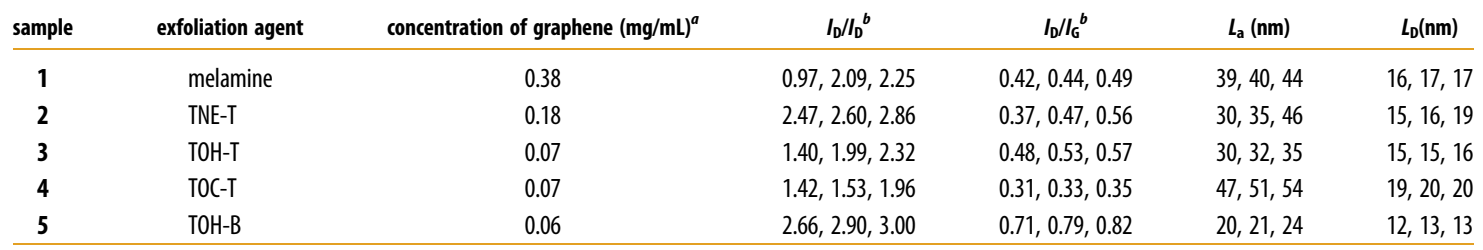

${ }^{a}$ Calculated by UV-vis-NIR absorption spectra. ${ }^{b}$ The different $I_{D} / I_{D}$ and $I_{D} / I_{G}$ values are from different locations in the sample.

fresh solvents, forming stable suspensions, which can be characterized by UV-vis-NIR absorption spectroscopy, showing Lambert-Beer behavior. Table 2 shows the final graphene concentrations of free exfoliating graphene dispersions in DMF calculated by UV - vis-NIR absorption (see Supporting Information for details, Figure S3).

In order to demonstrate the exfoliation of graphite and to analyze the quality of the produced graphene using the different exfoliating agents, fresh graphene dispersions of samples $\mathbf{1 - 5}$ were drop-cast onto silicon oxide surfaces and studied by Raman spectroscopy. This technique helps to identify graphene from graphite and few-layer graphene and has become a key technique to probe disorder in graphene through defect-activated peaks. ${ }^{22,23}$ Graphene exhibits $G$ and $2 D$ (also called $\mathrm{G}^{\prime}$ ) modes around 1580 and $2700 \mathrm{~cm}^{-1}$, respectively, that always satisfy the Raman selection rules. However, when graphene is affected by defects, the Raman features at 1345 (D band) and $1626 \mathrm{~cm}^{-1}$ ( $D^{\prime}$ band) appear in the spectrum. Recently, the evolution of the intensity ratio $I_{D} / I_{G}$ between the $G$ and the $D$ band has been used to provide a method to quantify the density of defects in monolayers and few-layer graphene..$^{24,25}$ Moreover, at moderate defect concentration, the $D^{\prime}$ peak can be clearly distinguished from the $G$ peak and the intensity ratio of the $D$ and $D^{\prime}$ peaks can be used to get information of the nature of the defects for a moderate amount of disorder. ${ }^{26}$

Figure 3 compares the $514 \mathrm{~nm}$ Raman spectra of bulk graphite and representative flakes from a DMF dispersion of samples $\mathbf{2}$ and $\mathbf{5}$. Even though the final concentrations of graphene are not as high as using melamine as exfoliating agent, Raman spectroscopy shows that the graphene produced in the different samples consists of few layers. The 2D band of the diverse samples is quite different from bulk graphite, it can be deconvoluted into four bands (Lorentzian peaks), which has been reported as a characteristic feature of the Raman spectra of bilayer graphene. ${ }^{27}$ In addition, Figure 4 shows a representation of the intensity ratio between $\mathrm{G}$ and $2 \mathrm{D}$ bands, and Raman spectra were taken in different areas of the sample. It has been described that the intensity of the $G$ band increases with the number of layers, ${ }^{28}$ thus from this figure, it seems that triaminotriazine derivatives produce better exfoliation of graphite.

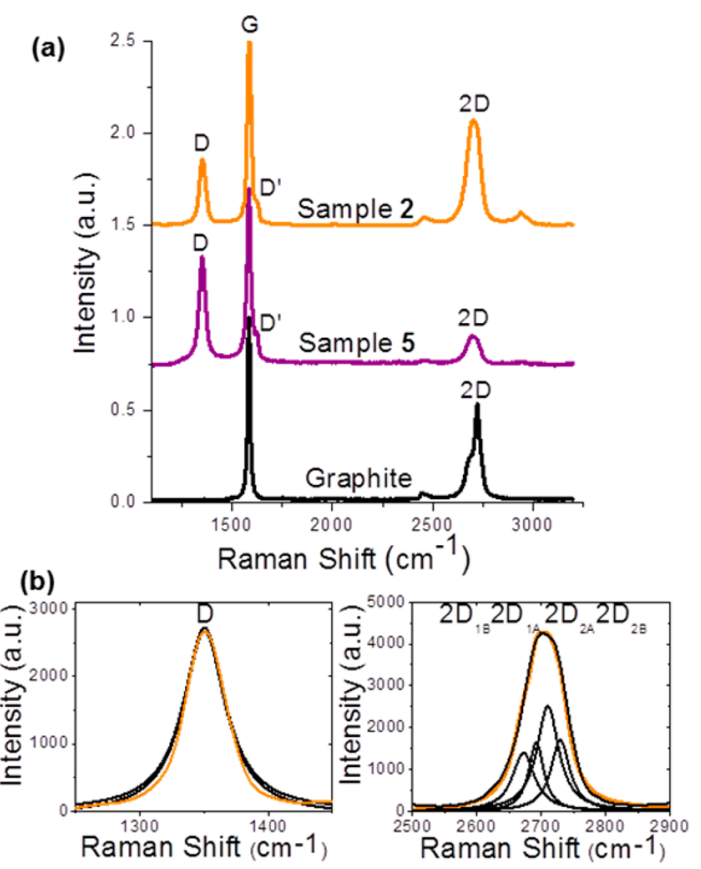

Figure 3. (a) Comparison of Raman spectra at $514 \mathrm{~nm}$ for graphite, sample 2, and sample 5. (b) D band in sample 2, fitted to one component. The four components of the 2D band in sample 2 .
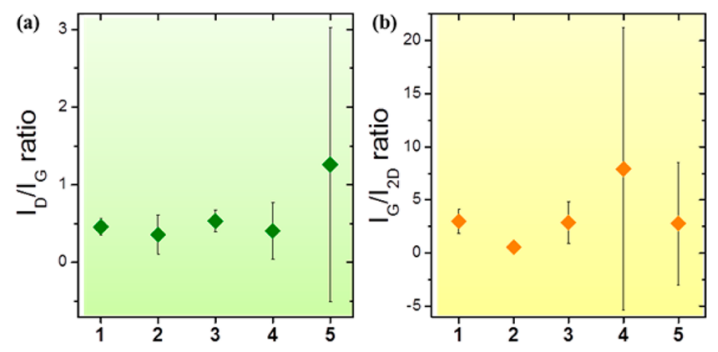

Figure 4. Statistical representation of intensity ratios between (a) $G$ and D bands and (b) $G$ and 2D bands in Raman spectra of samples 1-5. Data are taken from 10 different locations in the sample and are plotted with standard deviations.

We have also calculated the in-plane crystallite size $\left(L_{\mathrm{a}}\right)^{29}$ (amount of border with respect to the total crystallite area) and the distance between defects in the $\mathrm{sp}^{2}$ lattice $L_{\mathrm{D}}{ }^{24,30}$ Table 2 clearly shows smaller values for the benzene derivative, while similar data are observed for the triazine derivatives. The nature of these defects can be estimated by the intensity ratio of 


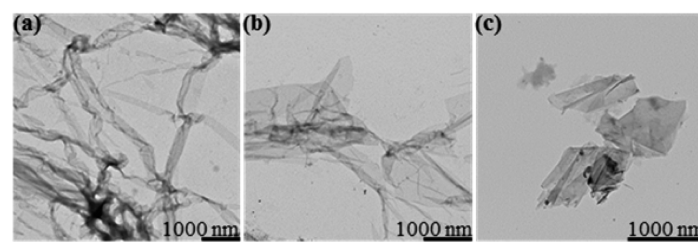

Figure 5. TEM images of graphene in (a) sample 1, (b) sample 3 , and (c) sample 5 .

the $D$ and $D^{\prime}$ peaks, using the model described by Eckmann et al. ${ }^{26}$ This ratio is maximum, around 13 , for $\mathrm{sp}^{3}$ defects, and it decreases to 7 for vacancy-like defects and reaches the minimum for boundary-like defects. Based on the data reported in Table 2 and Figure 4, these latter are the type of defects that we expect to find in our samples.

Graphene flakes can also be observed by transmission electron microscopy (TEM). Figure 5 shows representative images of graphene flakes, free from the exfoliating agents, produced by immersion of the respective dispersions onto a TEM grid. The flakes appear very large and extremely flexible, with many visible wrinkles.

Computations. In order to rationalize the experimental results, and especially to evaluate the relative ability of the studied compounds to exfoliate graphite and stabilize graphene, we performed computational calculations. Different factors must be taken into account: the structure of the individual components, the interactions between the absorbed molecules, and their affinity for the graphene surface.

Recently, several authors have performed theoretical studies on the interactions of small molecules and polycyclic aromatic compounds with graphene. ${ }^{31-35}$ The capability of tuning the graphene band structure is a hot topic, and the absorption of these molecules could be a simple and effective method to control the electronic properties of graphene systems.

From a theoretical point of view, the quantification and identification of the nature of interactions of adsorbed molecules on graphene present several challenges. One important aspect in this kind of system is the calculation of the dispersion interactions, which are ubiquitous weak attractive forces between molecules. It has become clear that they turn to be one of the dominant forces for large molecules and in supramolecular chemistry. The calculation of these dispersion forces is complex from a computational perspective. Quantum mechanical calculations based on density functional theory (DFT), with the most widely used exchange-correction DFT funtionals, that is, local density approximation (LDA $)^{36}$ and generalized gradient approximation (GGA), ${ }^{37,38}$ are often inadequate. Some studies have recently shown that the absorption strength of molecules on graphene is governed by dispersive interactions, ${ }^{33}$ and the omission of nonlocal electron correlations can strongly affect the calculated adsorption energy. A number of dispersion-corrected
DFT methods have been developed. The most widely used are probably the DFT-D family of methods by Grimme (mostly at the B97-D level) ${ }^{39}$ and fully nonlocal and computational expensive methods. ${ }^{40-42}$

In this paper, all the calculations were performed using GAUSSIAN $09^{43}$ suites of programs. In order to reduce the computational cost, we have preoptimized all the structures using the ONIOM ${ }^{44-46}$ method with $\mathrm{DFT}^{47}$ B3LYP/3-21G $\mathrm{G}^{* 48-50}$ and B97-D as the low- and high-theory levels, respectively. A graphene sheet consisting of 170 carbon atoms and 36 peripheral hydrogen atoms was used in the calculations, with $\mathrm{C}-\mathrm{C}$ and $\mathrm{C}-\mathrm{H}$ bond distances set at 1.46 and $1.01 \AA$, respectively. Preoptimized structures were fully optimized with a semilocal density functional with dispersion correction (B97-D) level of theory developed by Grimme. ${ }^{39}$ This method renders reasonable results at a very low computational cost in comparison to $\operatorname{CCSD}(\mathrm{T}),{ }^{51}$ and it has already proven to be useful in noncovalent interactions between graphene sheets. ${ }^{52}$ For the adsorbents, the molecular structures were fully optimized with B97-D method until the minima were localized or when the gradient convergence factor was better than $10^{-6}$ hartree/bohr. During the optimization steps, all species were free to move.

The absorption of different triazine and benzene derivatives on a graphene sheet has been studied. The geometry features of some of these compounds are depicted in Figure S5 (Supporting Information), showing the most stable conformation, in which the adsorbate atoms are located at the positions where the carbon atoms of the next second graphite-like sheet should be found, in agreement with similar studies. ${ }^{33}$ The adsorption energy, $E_{\text {ads }}$ for the exfoliating agents on the graphene sheet is defined according to the following equation:

$$
E_{\mathrm{ads}}=E_{\mathrm{Ea} / \text { Graphene }}-\left(E_{\mathrm{Ea}}+E_{\text {Graphene }}\right)
$$

where $E_{\mathrm{Ea} / \mathrm{Graphene}}$ is the energy of the system with the exfoliating agent $(\mathrm{Ea})$ adsorbed on graphene and $E_{\mathrm{Ea}}$ and $E_{\text {Graphene }}$ are the energies of the isolated exfoliating agent and graphene, respectively. With this definition, the adsorption energy is negative if the complete system is stabilized with the adsorption of the exfoliating agent on graphene.

Table 3 shows the adsorption energies of some triazine and benzene derivatives. These results are in concordance with previous computational studies. ${ }^{53}$ The stabilization energies of benzene and 1,3,5-triazine derivatives are similar, although slightly higher in the case of benzene rings (Table 3, entries 3, 8 and 4, 9). As expected, the presence of $\mathrm{NH}_{2}$ and $\mathrm{OH}$ groups in the aromatic core increases the adsorption energy, which is also higher with the number of groups, providing $\mathrm{NH}_{2}$ substituents with more stabilization than $\mathrm{OH}$ groups (Table 3, entries 4, 6 and 9, 11).

Close examination of the geometry features of the optimized structures reveals that the atoms of 
TABLE 3. Adsorption Energies ( $\left.E_{\text {ads }}\right)$ for Each Exfoliating Agent on the Graphene Sheet Calculated at B97-D Theory Level

\begin{tabular}{clc} 
entry & \multicolumn{1}{c}{ exfoliation agent } & $\boldsymbol{E}_{\text {ads }}{ }^{a}(\mathbf{k c a l} / \mathbf{m o l})$ \\
\hline 1 & 1,3,5-triazine & -13.70 \\
2 & 2-amino-1,3,5-triazine & -17.18 \\
3 & 2,4-diamino-1,3,5-triazine & -20.86 \\
4 & melamine & -24.34 \\
5 & 2,4,6-tri(N,N-dimethylamino)-1,3,5-triazine & -38.03 \\
6 & TOH-T & -18.75 \\
7 & benzene & -14.33 \\
8 & 1,3-diaminobenzene & -21.86 \\
9 & 1,3,5-triaminobenzene & -25.69 \\
10 & I,3,5-tri $(N, N$-dimethylamino)benzene & -39.28 \\
11 & TOH-B & -20.23 \\
\hline
\end{tabular}

${ }^{a}$ The adsorption energy for the exfoliating agents on the graphene sheet. (a)

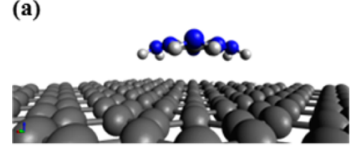

(c)

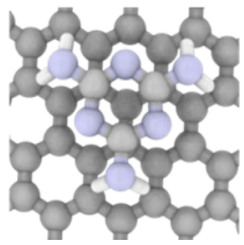

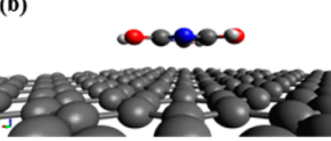

(d)

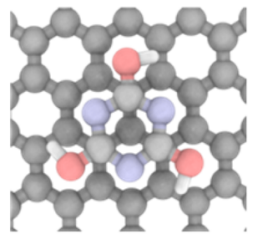

Figure 6. Calculated geometries of melamine adsorbed on a graphene sheet: (a) side view, (c) top view, and TOH-T adsorbed on a graphene sheet (b) side view, (d) top view.

hydrogen in the $\mathrm{NH}_{2}$ group are directed toward the graphene surface (Figure 6a). As previously reported, there is a great degree of interaction between the $\mathrm{NH}_{2}$ group and the graphene surface, which requires distortion of $\mathrm{NH}_{2}$ groups out of the triazine plane. In fact, the calculated distance from the graphene surface to the hydrogen atom of the $\mathrm{NH}_{2}(2.7 \AA)$ group in melamine is smaller than that for the hydrogen atom of the $\mathrm{OH}$ group in the 2,4,6-trihydroxy-1,3,5-triazine (3.1 A). It has been previously described ${ }^{53}$ that the adsorption energies of aminotriazines on graphene are directly related to the ability of these molecules to accept charge transfer from graphene to aminotriazines, in part through the presence of hydrogen atoms in the substituents. This fact explains why hydrogen atoms are directed toward the graphene surface and could also explain a higher adsorption energy for $\mathrm{NMe}_{2}$ substituents, which provides a larger number of hydrogen atoms.

However, these results do not agree with the experimental findings. As described above, the highest concentration of graphene was obtained using melamine as exfoliating agent and not using TNE-T. This last molecule has more hydrogen atoms that can interact with the graphene surface and, following this model, should present higher adsorption energy. This means
TABLE 4. Adsorption Energies ( $\left.E_{\text {ads }}\right)$ of Melamine and 1,3,5-Triaminobenzene Dimers on the Graphene Sheet Calculated at B97-D Theory Level

\begin{tabular}{clc} 
entry & \multicolumn{1}{c}{ exfoliation agent } & $\boldsymbol{E}_{\text {ads }}{ }^{\boldsymbol{a}}(\mathbf{k c a l} / \mathbf{m o l})$ \\
\hline 1 & melamine & -24.34 \\
2 & melamine dimer & -44.60 \\
3 & two melamine molecules & -44.94 \\
4 & $1,3,5$-triaminobenzene & -25.69 \\
5 & $1,3,5$-triaminobenzene dimer & -34.97 \\
6 & two 1,3,5-triaminobenzene molecules & -48.60 \\
\hline
\end{tabular}

${ }^{a}$ The adsorption energy for the exfoliating agents on the graphene sheet.

(a)
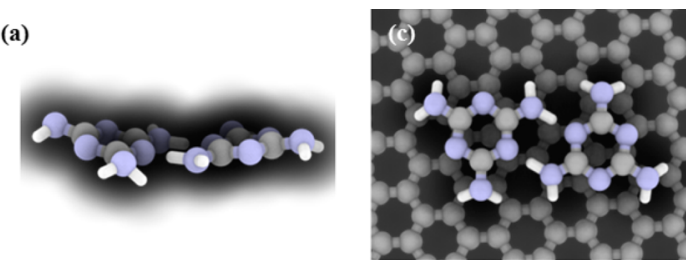

(b)
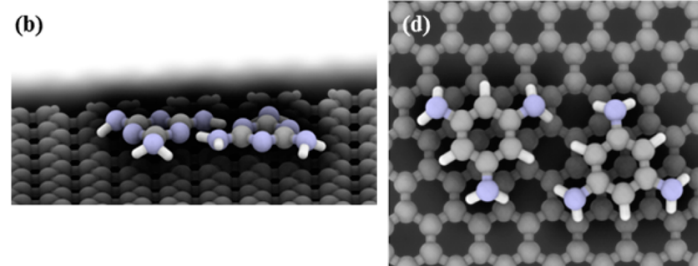

Figure 7. Calculated geometries of (a) melamine dimer, (b) melamine dimer on graphene side view, (c) melamine dimer on graphene top view, (d) 1,3,5-triaminobenzene dimer on graphene top view.

that the adsorption energy of discrete molecules on graphene is not sufficient by itself to justify the exfoliation and stabilization of graphite in good yields.

It is known that triaminotriazines can form hydrogen bonds that can create 2D molecular assemblies on different surfaces. ${ }^{54,55}$ Thus, we performed the computational calculations of these possible aggregations. The calculated results at B97-D level of theory for melamine and benzene derivative aggregates are collected in Table 4. The geometry features of some aggregates are depicted in Figure 7. It can be pointed out that the adsorption energy of the melamine dimer on the graphene surface is higher than the one of the 1,3,5-triamine benzene dimer. It is interesting to note that, in the case of melamine, this energy is similar when considering either a dimer or two individual molecules. Its behavior differs in the case of the benzene derivative (Table 4, entries 2, 3 and 5, 6). In the case of the benzene ring, the adsorption of two individual molecules is more favorable than the adsorption of the dimer.

Similar results were observed by Rochefort et al., ${ }^{56}$ who studied the adsorption of benzoic acids on graphene. Thus, whereas the absorption of one molecule of benzoic acid presents both $\mathrm{CO}$ and $\mathrm{OH}$ units in the $\mathrm{COOH}$ group bent toward the graphene sheet, the 


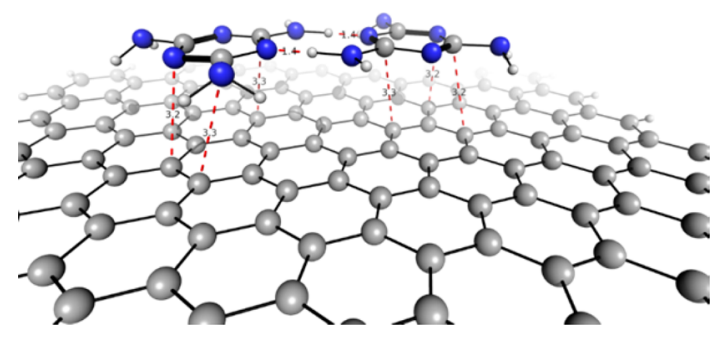

Figure 8. Melamine dimer on graphene.

$\mathrm{COOH}$ in the dimer remains parallel to the underlying surface. The energy of adsorption of the dimer was smaller than the energy of adsorption for two isolated molecules. The authors attributed this behavior to an increased $\pi-\pi$ repulsion. In our case, benzene derivatives do not form hydrogen bonds among themselves, while in the melamine dimer, the distance between two melamine molecules confirms the formation of hydrogen bonds. Moreover, the optimized structure of the dimer alone presents a 3D structure but, when it lays on graphene, forms a 2D structure by formation of hydrogen bonds on the graphene layer (Figure 8).

If we go further to a four melamine system, two geometrically different tetramers can be formulated, a linear one and a nonlinear one (Supporting Information, Figure S6). The two possible aggregation patterns have similar energy values, though the most favorable is the nonlinear one (Figure 9a). The adsorption of this $3 \mathrm{D}$ structure on a graphene sheet has also been studied (Supporting Information), following a similar behavior to the adsorption of the melamine dimer, in terms of stability (see above). Finally, in order to simulate the graphite exfoliation process, we placed the tetramers, both melamine and 1,3,5-triaminobenzene, inside two graphene layers (Supporting Information). In this case, due to the high molecular weight of this complex, the calculation was performed at $\mathrm{PM}^{57}$ theory level, which allows a higher number of atoms to be evaluated. Under these conditions, it can be observed that the melamine tetramer, from a $3 D$ pattern, becomes a $2 \mathrm{D}$ structure, while the graphene layers tend to separate (Figure 9b).

These results strongly suggest that the formation of a hydrogen-bonding network makes the formation of multipoint interactions with the surfaces of graphene possible and can be used for the exfoliation of graphite and the stabilization of graphene in different solvents.

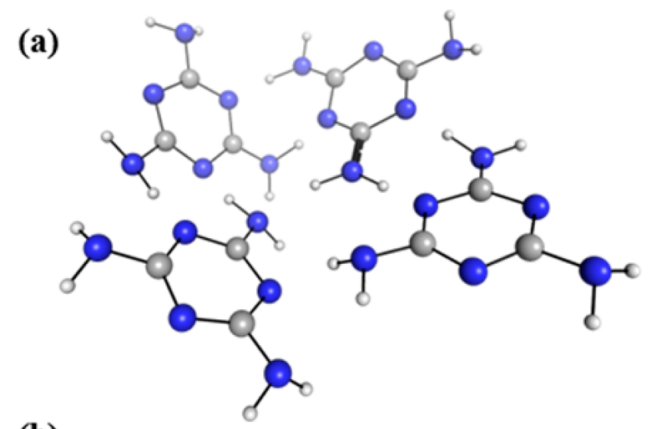

(b)

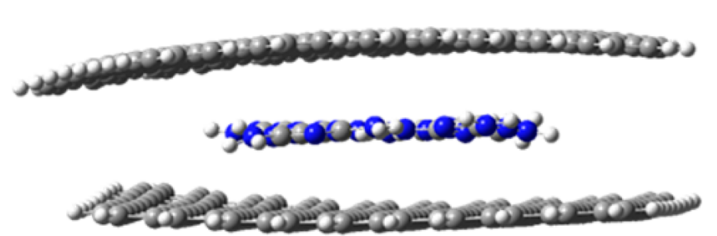

Figure 9. (a) Melamine tetramer, (b) melamine tetramer inside two graphene layers calculated at $\mathrm{PM}^{57}$ theory level.

These results provide also the basis for further experimental work for the preparation of noncovalently modified graphene, where derivatives of aminotriazines can be designed to form extensive hydrogen-bond 2D networks on the graphene surface with the aim of manipulating their electronic and chemical properties.

\section{CONCLUSIONS}

We have studied, both experimentally and theoretically, the interactions of a series of aromatic derivatives with graphene layers. We have found that, although melamine performs better in the exfoliation of graphite, triaminotriazine derivatives are also useful systems for the exfoliation and stabilization of graphene flakes in different solvents. The reason for this behavior can be attributed to two main features: (1) analogously to all the other investigated systems, melamine has an aromatic nucleus able to interact with the $\pi$-system of graphene; (2) in addition to this, melamine is able to form extended 2D networks, based on the presence of hydrogen bonds. These results can be usefully exploited toward the ideal system for the exfoliation of graphite, in which triaminotriazine derivatives can be designed to form extensive hydrogen-bond $2 \mathrm{D}$ networks on the graphene surface and, at the same time, manipulate their electronic and chemical properties by noncovalent interactions.

\section{MATERIALS AND METHODS}

Solvents were purchased from SDS and Fluka. Chemicals were purchased from Sigma-Aldrich and used as received without further purification. Graphite was purchased from Bay Carbon, Inc. (SP-1 graphite powder, www.baycarbon.com) and used without purification. The thermogravimetric analyses were performed with a TGA Q50 (TA Instruments) at $10^{\circ} \mathrm{C} / \mathrm{min}$ under nitrogen atmosphere. Raman spectra were recorded with an InVia Renishaw microspectrometer equipped with a $\mathrm{He}-\mathrm{Ne}$ laser. Raman samples were prepared from stable diluted dispersions of graphene/aromatic molecules by drop-casting of silicon oxide surfaces (Si-Mat silicon wafers, CZ). For the TEM analyses, concentrated dispersions of graphene/aromatic molecules were filtrated on a Millipore membrane (PTFE, $0.45 \mu \mathrm{m}$ ), 
paying special attention to keep the samples wet during the filtration processes. Graphene samples were redispersed in fresh water or DMF, forming stable dispersions. These dispersions were placed on a copper grid ( $3.00 \mathrm{~mm}, 200$ mesh), coated with carbon film by immersion, and dried under vacuum, and the sample was investigated by a TEM Philips EM 208 at an accelerating voltage of $100 \mathrm{kV}$. The milling treatments were carried out in a Retsch PM100 planetary mill at $100 \mathrm{rpm}$ during 30 min under air atmosphere.

Preparation of Graphene Dispersions. Graphite $(7.5 \mathrm{mg})$ and exfoliating agent $(0.16 \mathrm{mmol})$ were introduced in a stainless steel grinding bowl with 10 stainless steel balls ( $1 \mathrm{~cm}$ diameter). The bowl was closed and placed within the planetary mill. The ball-milling treatment conditions were $100 \mathrm{rpm}$ during $30 \mathrm{~min}$ under air atmosphere. After the treatment, the resulting solid mixture was suspended in $20 \mathrm{~mL}$ of water or DMF and sonicated for $1 \mathrm{~min}$. After letting the solutions rest for 5 days, the precipitate was removed, and the resulting dispersions were stable at room temperature for weeks.

Conflict of Interest: The authors declare no competing financial interest.

Acknowledgment. This work has been supported, in part, by the Spanish Ministerio de Economía y Competitividad (project CTQ2011-22410 and project IPT-2012-0429-420000), the University of Trieste and the Italian Ministry of Education MIUR (cofin Prot. 2010N3T9M4 and Firb RBAP11C58Y). A.M.R. would like to acknowledge MEC/MICINN for the FPU fellowship. Technical support from the High Performance Computing Service of University of Castilla-La Mancha and from Claudio Gamboz (University of Trieste, TEM images) is gratefully acknowledged.

Supporting Information Available: TGA of samples 1-5. Figures S1-S6, Tables S1 and S2, and Cartesian coordinates of all the stationary points (B97-D) and adsorption geometries discussed in the main text. This material is available free of charge via the Internet at http://pubs.acs.org.

\section{REFERENCES AND NOTES}

1. Palermo, V. Not a Molecule, Not a Polymer, Not a Substrate ... the Many Faces of Graphene as a Chemical Platform. Chem. Commun. 2013, 49, 2848-2857.

2. Novoselov, K. S.; Fal'ko, V. I.; Colombo, L.; Gellert, P. R.; Schwab, M. G.; Kim, K. A Roadmap for Graphene. Nature 2012, 490, 192-200.

3. Edwards, R. S.; Coleman, K. S. Graphene Synthesis: Relationship to Applications. Nanoscale 2013, 5, 38-51.

4. Wei, D.; Wu, B.; Guo, Y.; Yu, G.; Liu, Y. Controllable Chemical Vapor Deposition Growth of Few Layer Graphene for Electronic Devices. Acc. Chem. Res. 2013, 46, 106-115.

5. Mao, H. Y.; Laurent, S.; Chen, W.; Akhavan, O.; Imani, M.; Ashkarran, A. A.; Mahmoudi, M. Graphene: Promises, Facts, Opportunities, and Challenges in Nanomedicine. Chem. Rev. 2013, 113, 3407-3424.

6. Bianco, A. Graphene: Safe or Toxic? The Two Faces of the Medal. Angew. Chem., Int. Ed. 2013, 52, 4986-4997.

7. Bussy, C.; Ali-Boucetta, H.; Kostarelos, K. Safety Considerations for Graphene: Lessons Learnt from Carbon Nanotubes. Acc. Chem. Res. 2013, 46, 692-701.

8. Rodriguez-Perez, L.; Angeles Herranz, M.; Martin, N. The Chemistry of Pristine Graphene. Chem. Commun. 2013, 49, 3721-3735

9. Cui, X.; Zhang, C. Z.; Hao, R.; Hou, Y. L. Liquid-Phase Exfoliation, Functionalization and Applications of Graphene. Nanoscale 2011, 3, 2118-2126.

10. Coleman, J. N. Liquid Exfoliation of Defect-Free Graphene. Acc. Chem. Res. 2013, 46, 14-22.

11. Chen, D.; Feng, $\mathrm{H}_{\text {; }} \mathrm{Li}, \mathrm{J}$. Graphene Oxide: Preparation, Functionalization, and Electrochemical Applications. Chem. Rev. 2012, 112, 6027-6053.

12. Boukhvalov, D. W.; Katsnelson, M. I. Modeling of Graphite Oxide. J. Am. Chem. Soc. 2008, 130, 10697-10701.
13. Quintana, M.; Vazquez, E.; Prato, M. Organic Functionalization of Graphene in Dispersions. Acc. Chem. Res. 2013, 46, 138-148.

14. Georgakilas, V.; Otyepka, M.; Bourlinos, A. B.; Chandra, V.; Kim, N.; Kemp, K. C.; Hobza, P.; Zboril, R.; Kim, K. S. Functionalization of Graphene: Covalent and Non-covalent Approaches, Derivatives and Applications. Chem. Rev. 2012, 112, 6156-6214.

15. Parviz, D.; Das, S.; Ahmed, H. S. T.; Irin, F.; Bhattacharia, S.; Green, M. J. Dispersions of Non-covalently Functionalized Graphene with Minimal Stabilizer. ACS Nano 2012, 6, 8857-8867.

16. Kozlov, S. M.; Vines, F.; Goerling, A. Bandgap Engineering of Graphene by Physisorbed Adsorbates. Adv. Mater. 2011, 23, 2638.

17. Leon, V.; Quintana, M.; Antonia Herrero, M.; Fierro, J. L. G.; de la Hoz, A.; Prato, M.; Vazquez, E. Few-Layer Graphenes from Ball-Milling of Graphite with Melamine. Chem. Commun. 2011, 47, 10936-10938.

18. Vázquez, E.; Giacalone, F.; Prato, M. Non-conventional Methods and Media for the Activation and Manipulation of Carbon Nanoforms. Chem. Soc. Rev. 2013, 43, 58-69.

19. Jeon, I.-Y.; Choi, H.-J.; Jung, S.-M.; Seo, J.-M.; Kim, M.-J.; Dai, L.; Baek, J.-B. Large-Scale Production of Edge-Selectively Functionalized Graphene Nanoplatelets via Ball Milling and Their Use as Metal-Free Electrocatalysts for Oxygen Reduction Reaction. J. Am. Chem. Soc. 2013, 135, 1386-1393.

20. Kolesinska, B.; Kaminski, Z. J. The Umpolung of Substituent Effect in Nucleophilic Aromatic Substitution. A New Approach to the Synthesis of N,N-Disubstituted Melamines (Triazine Triskelions) under Mild Reaction Conditions. Tetrahedron 2009, 65, 3573-3576.

21. Marcano, D. C.; Kosynkin, D. V.; Berlin, J. M.; Sinitskii, A.; Sun, Z.; Slesarev, A.; Alemany, L. B.; Lu, W.; Tour, J. M. Improved Synthesis of Graphene Oxide. ACS Nano 2010, 4, 48064814.

22. Ferrari, A. C.; Basko, D. M. Raman Spectroscopy as a Versatile Tool for Studying the Properties of Graphene. Nat. Nanotechnol. 2013, 8, 235-246.

23. Dresselhaus, M. S.; Jorio, A.; Hofmann, M.; Dresselhaus, G.; Saito, R. Perspectives on Carbon Nanotubes and Graphene Raman Spectroscopy. Nano Lett. 2010, 10, 751-758.

24. Cancado, L. G.; Jorio, A.; Martins Ferreira, E. H.; Stavale, F.; Achete, C. A.; Capaz, R. B.; Moutinho, M. V. O.; Lombardo, A.; Kulmala, T. S.; Ferrari, A. C. Quantifying Defects in Graphene via Raman Spectroscopy at Different Excitation Energies. Nano Lett. 2011, 11, 3190-3196.

25. Jorio, A.; Lucchese, M. M.; Stavale, F.; Martins Ferreira, E. H.; Moutinho, M. V. O.; Capaz, R. B.; Achete, C. A. Raman Study of Ion-Induced Defects in N-Layer Graphene. J. Phys.: Condens. Matter 2010, 22, 334204.

26. Eckmann, A.; Felten, A.; Mishchenko, A.; Britnell, L.; Krupke, R.; Novoselov, K. S.; Casiraghi, C. Probing the Nature of Defects in Graphene by Raman Spectroscopy. Nano Lett. 2012, 12, 3925-3930.

27. Cancado, L. G.; Reina, A.; Kong, J.; Dresselhaus, M. S. Geometrical Approach for the Study of $G^{\prime}$ Band in the Raman Spectrum of Monolayer Graphene, Bilayer Graphene, and Bulk Graphite. Phys. Rev. B 2008, 77, 245408245417.

28. Xu, M.; Fujita, D.; Gao, J.; Hanagata, N. Auger Electron Spectroscopy: A Rational Method for Determining Thickness of Graphene Films. ACS Nano 2010, 4, 2937-2945.

29. Cancado, L. G.; Takai, K.; Enoki, T.; Endo, M.; Kim, Y. A.; Mizusaki, H.; Jorio, A.; Coelho, L. N.; Magalhaes-Paniago, R.; Pimenta, M. A. General Equation for the Determination of the Crystallite Size $L_{a}$ of Nanographite by Raman Spectroscopy. Appl. Phys. Lett. 2006, 88, 163106.

30. Lucchese, M. M.; Stavale, F.; Ferreira, E. H. M.; Vilani, C.; Moutinho, M. V. O.; Capaz, R. B.; Achete, C. A.; Jorio, A. Quantifying Ion-Induced Defects and Raman Relaxation Length in Graphene. Carbon 2010, 48, 1592-1597.

31. Lu, Y. H.; Chen, W.; Feng, Y. P.; He, P. M. Tuning the Electronic Structure of Graphene by an Organic Molecule. J. Phys. Chem. B 2009, 113, 2-5. 
32. Zhang, Y.-H.; Zhou, K.-G.; Xie, K.-F.; Zeng, J.; Zhang, H.-L.; Peng, Y. Tuning the Electronic Structure and Transport Properties of Graphene by Noncovalent Functionalization: Effects of Organic Donor, Acceptor and Metal Atoms. Nanotechnology 2010, 21, 065201.

33. Kozlov, S. M.; Vines, F.; Goerling, A. On the Interaction of Polycyclic Aromatic Compounds with Graphene. Carbon 2012, 50, 2482-2492.

34. Lazar, P.; Karlicky, F.; Jurecka, P.; Kocman, M.; Otyepkova, E.; Safarova, K.; Otyepka, M. Adsorption of Small Organic Molecules on Graphene. J. Am. Chem. Soc. 2013, 135, 6372-6377.

35. Brunetti, F. G.; Isla, H.; Arago, J.; Orti, E.; Perez, E. M.; Martin, N. Exploiting Multivalent Nanoparticles for the Supramolecular Functionalization of Graphene with a Nonplanar Recognition Motif. Chem.-Eur. J. 2013, 19, 9843-9848.

36. Becke, A. D. Density-Functional Exchange-Energy Approximation with Correct Asymptotic-Behavior. Phys. Rev. A 1988, 38, 3098-3100.

37. Burke, K. Perspective on Density Functional Theory. J. Chem. Phys. 2012, 136, 150901.

38. Cohen, A. J.; Mori-Sanchez, P.; Yang, W. Challenges for Density Functional Theory. Chem. Rev. 2012, 112, 289-320.

39. Grimme, S. Semiempirical GGA-Type Density Functional Constructed with a Long-Range Dispersion Correction. J. Comput. Chem. 2006, 27, 1787-1799.

40. Dion, M.; Rydberg, H.; Schroder, E.; Langreth, D. C.; Lundqvist, B. I. van der Waals Density Functional for General Geometries. Phys. Rev. Lett. 2004, 92, 246401.

41. Eshuis, H.; Bates, J. E.; Furche, F. Electron Correlation Methods Based on the Random Phase Approximation. Theor. Chem. Acc. 2012, 131, 1084.

42. Ren, X.; Rinke, P.; Joas, C.; Scheffler, M. Random-Phase Approximation and Its Applications in Computational Chemistry and Materials Science. J. Mater. Sci. 2012, 47, 7447-7471.

43. Frisch, M. J.; Trucks, G. W.; Schlegel, H. B.; Scuseria, G. E.; Robb, M. A.; Cheeseman, J. R.; Scalmani, G.; Barone, V.; Mennucci, B.; Petersson, G. A.; et al. Gaussian 09, revision C; Gaussian, Inc.: Wallingford, CT, 2009.

44. Vreven, T.; Morokuma, K. On the Application of the IMOMO (Integrated Molecular Orbital Plus Molecular Orbital) Method. J. Comput. Chem. 2000, 21, 1419-1432.

45. Vreven, T.; Morokuma, K.; Farkas, O.; Schlegel, H. B.; Frisch, M. J. Geometry Optimization with QM/MM, ONIOM, and Other Combined Methods. I. Microiterations and Constraints. J. Comput. Chem. 2003, 24, 760-769.

46. Senn, H. M.; Thiel, W. QM/MM Methods for Biomolecular Systems. Angew. Chem., Int. Ed. 2009, 48, 1198-1229.

47. Parr, R. G.; Yang, W. Density-Functional Theory of Atoms and Molecules; Oxford University Press: New York, 1994.

48. Kohn, W.; Becke, A. D.; Parr, R. G. Density Functional Theory of Electronic Structure. J. Phys. Chem. 1996, 100, 12974-12980.

49. Ditchfie, R.; Hehre, W. J.; Pople, J. A. Self-Consistent Molecular-Orbital Methods. Extended Gaussian-Type Basis for Molecular-Orbital Studies of Organic Molecules. J. Chem. Phys. 1971, 54, 724.

50. Hehre, W. J.; Ditchfie, R.; Pople, J. A. Self-Consistent Molecular-Orbital Methods. Futher Extensions of GaussianType Basis Sets for Use in Molecular-Orbital Studies of Organic-Molecules. J. Chem. Phys. 1972, 56, 2257.

51. Scuseria, G. E.; Janssen, C. L.; Schaefer, H. F. An Efficient Reformulation of the Closed-Shell Coupled Cluster Single and Double Excitation (CCSD) Equations. J. Chem. Phys. 1988, 89, 7382-7387.

52. Grimme, S.; Muck-Lichtenfeld, C.; Antony, J. Noncovalent Interactions between Graphene Sheets and in Multishell (Hyper) Fullerenes. J. Phys. Chem. C 2007, 111, 11199-11207.

53. Wuest, J. D.; Rochefort, A. Strong Adsorption of Aminotriazines on Graphene. Chem. Commun. 2010, 46, 29232925.

54. Zhang, X.; Chen, T.; Chen, Q.; Wang, L.; Wan, L.-J. SelfAssembly and Aggregation of Melamine and MelamineUric/Cyanuric Acid Investigated by STM and AFM on Solid Surfaces. Phys. Chem. Chem. Phys. 2009, 11, 7708-7712.
55. Llanes-Pallas, A.; Palma, C.-A.; Piot, L.; Belbakra, A.; Listorti, A.; Prato, M.; Samori, P.; Armaroli, N.; Bonifazi, D. Engineering of Supramolecular H-Bonded Nanopolygons via SelfAssembly of Programmed Molecular Modules. J. Am. Chem. Soc. 2009, 131, 509-520.

56. Rochefort, A.; Wuest, J. D. Interaction of Substituted Aromatic Compounds with Graphene. Langmuir 2009, 25, 210-215.

57. Stewart, J. J. P. Optimization of Parameters for Semiempirical Methods V: Modification of NDDO Approximations and Application to 70 Elements. J. Mol. Model. 2007, 13, 1173-1213. 